Kai Starke

\title{
Magnetic Dichroism in Core-Level Photoemission
}

With 64 Figures 


\section{Contents}

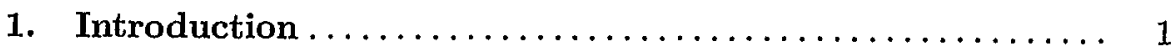

2. Theoretical Considerations $\ldots \ldots \ldots \ldots \ldots \ldots \ldots \ldots \ldots, 3$

2.1 Atomic Multiplet Theory ....................... 4

2.1.1 Selection Rules and Total Final States ........... 4

2.1.2 Photoemission Final States and Line Strength ...... 8

2.2 Simple Cases............................. 10

2.2.1 Gd 4f Photoemission: The Role of Final-State

Spin-Orbit Interaction .................... 10

2.2.2 Tb 4 f Photoemission: The ${ }^{8} \mathrm{~S}_{7 / 2}$ Component ........ 13

2.3 Geometries for Magnetic Dichroism .................. 14

2.3.1 Circularly Polarized Light $\ldots \ldots \ldots \ldots \ldots \ldots \ldots \ldots, 16$

2.3.2 Linearly Polarized Light................... 18

2.4 Binding Energy and Chemical Shift ................ 19

2.4.1 Core-Level Binding Energy in Rare-Earth Metals ..... 20

2.4.2 4f Photoemission and Surface Core-Level Shift ...... 25

3. Experimental Techniques $\ldots \ldots \ldots \ldots \ldots \ldots \ldots \ldots \ldots \ldots \ldots$

3.1 Sample Preparation.......................... 29

3.1.1 Rare-Earth-Metal Evaporators ............... 30

3.1.2 Wide-Temperature-Range Tungsten-Crystal Holder ... 31

3.1.3 Structural and Magnetic Characterization of Rare-Earth-Metal Films................... 33

3.2 X-Ray Magnetic Circular Dichroism as a Complementary Method................... 37

3.3 Beamlines for Circularly Polarized Soft X-Rays ......... 39

4. Closed Shells ............................. 43

4.1 Analytical Single-Particle Model.................... 44

4.2 Transition Metals: Fe $2 p$ and Fe $3 p \ldots \ldots \ldots \ldots \ldots \ldots \ldots, 45$

4.3 Rare-Earth Metals: Gd $4 d \ldots \ldots \ldots \ldots \ldots \ldots \ldots \ldots \ldots .50$ 
5. Rare-Earth 4f Shells ......................... 55

5.1 Gd Metal............................... 55

5.2 Heavier Rare-Earth Metals: $\mathrm{Tb}$ and $\mathrm{Dy} \ldots \ldots \ldots \ldots \ldots \ldots, 57$

5.3 Intermetallic Compounds: $\mathrm{TbFe}_{x} \ldots \ldots \ldots \ldots \ldots \ldots \ldots$

6. Magnetic Circular Dichroism in Resonant Photoemission . 65

6.1 A Primer on Fano's Theory $\ldots \ldots \ldots \ldots \ldots \ldots \ldots \ldots 66$

$6.2 \mathrm{Gd} 4 \mathrm{~d}-4 \mathrm{f}$ Giant Resonance $\ldots \ldots \ldots \ldots \ldots \ldots \ldots \ldots \ldots . \ldots 69$

6.2.1 Identification of X-Ray Absorption Lines .......... 71

6.2.2 Magnetic Cicular Dichroism in Resonant 4d-4f Photoemission.............. 73

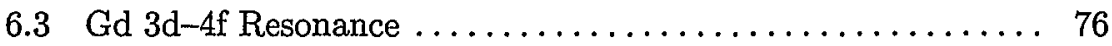

6.3.1 High-Resolution X-Ray Absorption Magnetic Circular Dichroism ...................... 77

6.3.2 Spin Flip in Resonant Photoemission .......... 79

7. Magnetic Dichroism in Photoemission

as a Near-Surface Magnetic Probe ................ 85

7.1 Magnetic Surface Reconstruction $\ldots \ldots \ldots \ldots \ldots \ldots \ldots \ldots . \ldots 6$

$7.1 .1 \mathrm{~Tb}(0001) \ldots \ldots \ldots \ldots \ldots \ldots \ldots \ldots \ldots \ldots \ldots, 89$

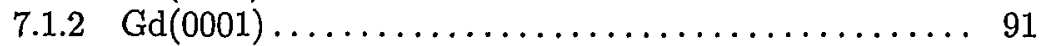

7.2 Atomic-Layer Resolution at Heteromagnetic Interfaces:

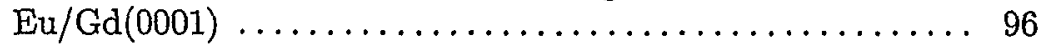

7.2.1 An Atomically Sharp Rare-Earth Interface......... 96

7.2.2 Layer-Dependent Magnetic Coupling of Ordered Eu Layers ............................. 100

7.2.3 Thicker Eu Films on Gd(0001) .............. 107

8. Conclusions and Future Directions ............... 111

A. Angle-Resolved Photoemission from f Shells: Gd ....... 115

References ................................. 121

Glossary of Acronyms $\ldots \ldots \ldots \ldots \ldots \ldots \ldots \ldots \ldots \ldots \ldots \ldots \ldots$

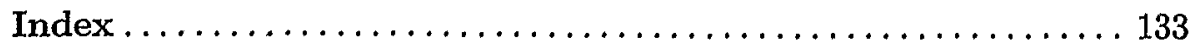

